\title{
Matter-Bounce Spin-Cosmology and consistency with BICEP2 data
}

\author{
Antonino Marciano* \\ Department of Physics, Fudan University \\ E-mail: marciano@fudan.edu.cn
}

\begin{abstract}
A nonsingular bouncing cosmology can be achieved by introducing a fermion field with a condensation occurring at high energy scales. The gap energy density restored in the regular state of a cosmic fermion can yield a short period of ekpyrotic phase in a contracting universe. The unwanted primordial anisotropies can be diluted during the ekpyrotic contraction. To derive a nearly scale-invariant CMB spectrum, another matter field is required. We deepen one possible curvaton mechanism by involving one another fermion field without condensation and of which the mass is lighter than the background field. By virtue of the fermion curvaton mechanism, this model is consistent with the latest cosmological data.
\end{abstract}

Frontiers of Fundamental Physics 14 - FFP14,

15-18 July 2014

Aix Marseille University (AMU) Saint-Charles Campus, Marseille

\footnotetext{
*Speaker.
} 